\title{
BRST Charge and Poisson Algebras ${ }^{\dagger}$
}

\author{
H. Caprasse
}

Département d'Astrophysique, Université de Liège, Institut de Physique B-5, Allée du 6 aout, Sart-Tilman B-4000, Liege, Belgium

E-Mail: caprasse@vm1.ulg.ac.be

\begin{abstract}
An elementary introduction to the classical version of gauge theories is made. The shortcomings of the usual gauge fixing process are pointed out. They justify the need to replace it by a global symmetry: the BRST symmetry and its associated BRST charge. The main mathematical steps required to construct it are described. The algebra of constraints is, in general, a nonlinear Poisson algebra. In the nonlinear case the computation of the BRST charge by hand is hard. It is explained how this computation can be made algorithmic. The main features of a recently created BRST computer algebra program are described. It can handle quadratic algebras very easily. Its capability to compute the BRST charge as a formal power series in the generic case of a cubic algebra is illustrated.
\end{abstract}

Keywords: guage theory, BRST symmetry

\section{Introduction}

Gauge invariance plays a key role in the formulation of all basic physical theories: this is true both at the classical and quantum levels. General relativity as well as all field theoretic models which describe the electromagnetic, the weak and the strong interactions are based on it. One feature of gauge invariant theories is that they contain what physicists call 'non-observable' quantities. These are objects which are not in one-to-one correspondance with physical measurements. This means that there are several ways to express them as functions of the phase space variables. A way to eliminate this arbitrariness is to introduce constraints among the phase space variables themselves. One says that one fixes the gauge. However, it turns out that this procedure almost always destroys both locality and covariance of the theory. On the other hand, this choice of gauge is a local operation, and one would like, of course, such a fixing of gauge to be global. Therefore, one needs to find something else to express the gauge arbitrariness and to single out physical quantities. In Becchi et al. [1] and Tyutin [2] and many subsequent works (see, for instance, Henneaux and Teitelboim [3] and the references therein), it has been shown that, if one works in a properly defined extended phase space, all gauge theories possess a nice symmetry called the BRST symmetry. Its generator is the BRST charge. This symmetry is global, and its use is sufficient to express gauge invariance in a proper way. It allows us to properly identify the space of physical states. BRST symmetry turns out to be essential in the quantization of Yang-Mills theory and in the theory of strings.

${ }^{\dagger}$ Talk given at the MEDICIS thematic workshop on 'Lie Computations', CIRM, Marseille, France, 28th November 1994 1365-8050 (C) 1997 Chapman \& Hall 
The work presented here is relevant to the classical formulation of the BRST symmetry. Considering classical gauge theories for which the (first class) constraints obey a linear as well as a nonlinear Poisson algebra, it is shown that the computation of the BRST charge can be made algorithmic. Its implementation in Reduce is discussed, and its capabilities illustrated. This work has been done in collaboration with Burnel and Dresse, and is described in Burnel et al. [4].

In Sect. 2, an elementary (and grossly oversimplified) introduction to gauge theories is made. The need to introduce the BRST symmetry is explained. In Sect. 3, the main mathematical steps which lead to BRST symmetry are reviewed. These use homological algebra extensively. In Sect. 4, the algorithm to compute the BRST charge is described. Finally, in Sect. 5, a few caracteristics of its implementation in Reduce are considered. Its capabilities are illustrated through the consideration of the case where the constraints are characterized by a cubic Poisson algebra.

\section{Introduction to Gauge Theories}

\subsection{Lagrangian Formulation}

In classical mechanics, one describes a physical system from the action principle:

$$
\begin{aligned}
S & =\int_{t_{1}}^{t_{2}} \mathcal{L}\left(q^{i}, \dot{q}^{i}\right) d \tau \\
\delta S & =0
\end{aligned}
$$

$S$ is the action, $t_{1}, t_{2}$ are the initial and final times, $\mathcal{L}$ is the Lagrangian, $q^{i}(i=1 \ldots n)$ are the coordinates and $\dot{q}^{i}$ are their time derivatives.

The corresponding dynamical equations are:

$$
\frac{d}{d t}\left(\frac{\partial \mathcal{L}}{\partial \dot{q}^{i}}\right)-\frac{\partial \mathcal{L}}{\partial q^{i}}
$$

Once the initial conditions $q^{i}(0), \dot{q}^{i}(0)$ are given, they allow to determine $q^{i}(t), \dot{q}^{i}(t)$ for any $t$ so that the physical state of the system is uniquely determined. However, the converse is not true, in general.

A given physical state may be described by several (generalized) coordinate variables. For instance let:

$$
\mathcal{L} \equiv \mathcal{L}\left(q^{1}-q^{2}, \dot{q}^{1}-\dot{q}^{2}\right)
$$

All functions $q^{i}(t), i=1,2$

$$
q^{i}(t)=q_{0}^{i}(t)+\gamma(t)
$$

where $\gamma(t)$ is an arbitrary function of $t$ such that $\gamma(0)=0$ describe the same physical state. So, an intuitive way to see gauge theories is to think of them as models which describe a physical system through a set of generalized coordinate variables which are not uniquely determined by the dynamical equations and the initial conditions. 


\subsection{Hamiltonian Formulation}

Momenta are defined by

$$
p_{i}=\frac{\partial \mathcal{L}}{\partial \dot{q}^{i}}=f\left(q^{i}, \dot{q}^{i}\right)
$$

Together with the coordinates they form the phase space of the system. If we express the total time derivative in (3) in terms of the partial derivatives, one sees that the accelerations $\ddot{q}^{i}$ are uniquely defined only if

$$
\operatorname{dtm}\left(\frac{\partial^{2} \mathcal{L}}{\partial \dot{q}^{i} \partial \dot{q}^{j}}\right) \neq 0
$$

Here one is interested to the case where this determinant is equal to zero. In that case, the functions given by (6) cannot be inverted. The momenta are not all independent, i.e. there exists $K$ relations among the $q^{i \prime} s, p_{i}{ }^{\prime} s$. Let them be

$$
C_{m}\left(q^{i}, p_{i}\right)=0, m=1, \ldots K
$$

These relations mean that the motion of the system develops inside a subspace of the phase space. One can give a hamiltonian formulation of the action principle. When such relations exist, the hamiltonian $(H)$ is not uniquely determined. Indeed, one can always replace it by

$$
H \rightarrow H^{\prime}=H+u^{m}(p, q) C_{m}
$$

The eqations of motion can be rewritten

$$
\begin{aligned}
& \dot{q}^{i}=\frac{\partial H}{\partial p_{i}}+u^{m} \frac{\partial C_{m}}{\partial p_{i}} \\
& \dot{p}_{i}=-\frac{\partial H}{\partial q^{i}}-u^{m} \frac{\partial C_{m}}{\partial q^{i}} \\
& C_{m}(q, p)=0
\end{aligned}
$$

The constraints should be conserved in time. This means

$$
\dot{C}_{m}=\left\{C_{m}, H\right\}+u^{m^{\prime}}\left\{C_{m}, C_{m^{\prime}}\right\}=0
$$

where, by definition, the Poisson brackets are

$$
\{A, B\}=\frac{\partial A}{\partial q^{i}} \frac{\partial B}{\partial p_{i}}-\frac{\partial A}{\partial p_{i}} \frac{\partial B}{\partial q^{i}}
$$

New constraints are generated by (13). Some of them depend on the $u$ 's. They are such that some of their Poisson brackets with the $C_{m}$ 's are different from zero. When this is encountered, one can eliminate some of the Lagrange parameters in (9). They are called second class constraints. They can be treated properly by replacing the Poisson brackets by the Dirac brackets [5]. So as not to obscure the discussion, one makes the simplifying assumption that all new constraints are independent of the $u$ 's. If there are $J$ 
new constraints, they must be added to the previous one. They are called first class constraints. All these new constraints are first class functions. This means that they satisfy

$$
\left\{F, C_{i}\right\}=\alpha_{i}^{j}(p, q) C_{j}
$$

One groups them together with the primary constraints in $M=K+J$ constraints. They form a Poisson algebra, i.e. they satisfy the equation

$$
\left\{C_{a}, C_{b}\right\}=f_{a b}{ }^{c}\left(q^{i}, p_{i}\right) C_{c}
$$

Each first class constraint is also the generator of a gauge transformation which acts on any function $F$ of the phase space variables in the following way:

$$
\delta_{\epsilon} F=\epsilon^{a}\left\{F, C_{a}\right\}
$$

These transformations determine the gauge orbits.

\subsection{The Algebraic Structures on Phase Space}

Let $C^{\infty}(P)$ be the set of regular functions on phase space such that

(i) multiplication is associative;

(ii) Poisson brackets of any two functions exists and have the derivation property.

The geometry of phase space is sympletic.

Let $\Sigma$ be the surface in phase space defined by all first class functions. One defines:

$$
N(P)=\left\{F \mid F=\lambda^{a}(q, p) C_{a}\right\}
$$

This set is invariant under the gauge transformations (17). Moreover, it is an ideal of $C^{\infty}(P)$. One is led to define the set of regular functions on $\Sigma$, which is named $C^{\infty}(\Sigma)$, as the quotient

$$
C^{\infty}(\Sigma)=C^{\infty}(P) / N(P)
$$

Two functions $F$ and $F^{\prime}$ are said equivalent, i.e.

$$
F \approx F^{\prime}
$$

iff

$$
F^{\prime}-F=\lambda^{a}(q, p) C_{a}
$$

A quantity $\Phi$ is physical iff

- it belongs to $C^{\infty}(\Sigma)$;

- it is invariant under all transformations (17). 


\subsection{Gauge Fixing}

The gauge freedom indicates that there are several sets of canonical variables that correspond to a given physical state. Each physical state is characterized by an equivalence class in $C^{\infty}(\Sigma)$. One would like to recover the one-to-one correspondance between it and its expression in terms of the canonical variables. This is only possible if one fixes the gauge. This can be done if one imposes additional relations

$$
\left\{f_{b}(q, p) \approx 0 \quad b=1, \ldots, N\right\}
$$

chosen in such a way that

$$
\delta \epsilon^{a}\left\{f_{b}, C_{a}\right\} \approx 0 \Rightarrow \delta \epsilon^{a}=0
$$

This obviously implies that

(i) $N=M$.

(ii) $\operatorname{dtm}\left(\left\{f_{b}, C_{a}\right\}\right) \neq 0$.

The surface generated by (20) (with $N=M$ ) should intersect gauge orbits at one point only. If this is always possible locally, it turns out that it is a requirement impossible to satisfy globally. In particular, this is always so for non-abelian gauge theories. There are also other problems with this procedure: locality and (or) covariance of the theory are lost. A celebrated example of this is the Coulomb gauge $(\operatorname{div} \vec{A}=0)$ in electrodynamics. All this justifies the need to replace gauge fixing by something else: this is BRST symmetry. Its nice properties are:

- it is a global symmetry,

- it avoids the construction of the reduced phase space, and

- it preserves locality and covariance.

The price one has to pay is the necessity to enlarge the original phase space. Let us mention that a very good account of the history of its discovery can be found in Hooft [6]

\section{Construction of the BRST Charge}

The most direct way to introduce BRST symmetry is to use homological algebra. A detailed covering of its deduction can be found in Henneaux and Teitelboim [3]. In this short introduction, one can only review the main mathematical steps.

\subsection{Phase Space Extension}

To each first class constraint $C_{a}$ one associates additional 'fermionic variables' $\eta^{a}$ and $\mathcal{P}_{a}$. They are odd parity Grassmann variables. $\eta^{a}$ has ghost number 1 while $\mathcal{P}_{a}$ has ghost number -1 (these last variables are called antighosts). Poisson brackets are generalized to these variables adding to (14) the new contribution

$$
\{A, B\}_{g h}=-\left(\frac{A \overleftarrow{\partial}}{\partial \eta^{a}} \frac{\vec{\partial} B}{\partial \mathcal{P}_{a}}+\frac{A \overleftarrow{\partial}}{\partial \mathcal{P}_{a}} \frac{\vec{\partial} B}{\partial \eta^{a}}\right)
$$


In particular, one gets:

$$
\left\{\eta^{a}, \eta^{b}\right\}=\left\{\mathcal{P}_{a}, \mathcal{P}_{b}\right\}=0, \quad\left\{\eta^{a}, \mathcal{P}_{b}\right\}=-\delta_{b}^{a} .
$$

Phase space is therefore enlarged:

$$
\left(q^{i}, p_{j}\right) \quad \rightarrow \quad\left(q^{i}, p_{j}, \eta^{a}, \mathcal{P}_{a}\right)
$$

Once this is done, one can extend the hamiltonian formulation to systems involving Grassmann variables and constraints involving them.

\subsection{Mathematical Ingredients}

One has to use several aspects of graded differential algebras. One introduces the graded algebra $A$

$$
\begin{aligned}
& A=\underset{k}{\oplus} A_{k} \\
& A_{i} A_{j} \subset A_{i+j}
\end{aligned}
$$

which contains all polynomials one can build on the enlarged phase space. $k$ is the grading - it is not necessarily positive. There are several ways to define it, as we shall see. If $x \in A_{i}$ then the degree of $x$ is

$$
\operatorname{deg} x=i
$$

In this frame, one introduces the notion of differential. $D$ is a differential if

- $D(x y)=x D(y)+(-1)^{\epsilon_{D} \epsilon_{y}}(D(x)) y, \quad \forall x, y \in A$,

- $\mathcal{D}=\operatorname{deg} D(x)-\operatorname{deg} x= \pm 1$,

- $D^{2}=0$,

- $\epsilon(D)=1$.

$\epsilon$ in the last equation means the parity of $D$. It is 0 or 1 for any object. This is the deg of $D$ with respect to a $Z_{2}$ grading applied on $A$. In concrete terms, on can say that it distinguises between quantities of 'Bose' and 'Fermi' types. The kernel and the image of $D$ are defined by

$$
\begin{aligned}
\operatorname{Ker} D & =\{x \mid D x=0\} \\
\operatorname{Im} D & =\{x \mid \exists y \in A: D y=x\}
\end{aligned}
$$

Then for all elements of $A$ of degree $k$ one can define the cohomology (homology):

$$
\begin{aligned}
H^{k}(D) & =\frac{(\operatorname{Ker} D)^{k}}{(\operatorname{Im} D)^{k}} \text { if } d=1 \\
H_{k}(D) & =\frac{(\operatorname{Ker} D)^{k}}{(\operatorname{Im} D)^{k}} \text { if } d=-1
\end{aligned}
$$

since $\operatorname{Im} D$ is an ideal of $\operatorname{Ker} D$. 
Finally, one uses the concept of contracting homotopy $\sigma$. Suppose that for a certain grading of $A$ an operator $\Lambda$ exists, such that

$$
\begin{aligned}
& A=\underset{\lambda}{\oplus} A_{\lambda} \\
& \Lambda x_{\lambda}=\lambda x_{\lambda} \\
& \sigma D+D \sigma=\Lambda(\text { we suppose } \mathcal{D}=1)
\end{aligned}
$$

then

$$
\begin{aligned}
& H^{0}(D) \neq 0 \\
& H^{\lambda}(D)=0 \forall \lambda \neq 0
\end{aligned}
$$

The reason why $H^{\lambda}(D)$ is 0 comes from the fact that for $\lambda \neq 0$ one gets, successively,

$$
x_{\lambda}=\frac{1}{\lambda} \Lambda x_{\lambda}=\frac{1}{\lambda}(\sigma D+D \sigma) x_{\lambda}=D\left(\frac{1}{\lambda} \sigma x_{\lambda}\right)
$$

which shows that any element which belongs to the kernel of $D$ also belongs to its image (here $y=\frac{1}{\lambda} \sigma x_{\lambda}$ ).

\subsection{BRST Symmetry}

First, taking for the grading the degree in the $\mathcal{P}_{a}$ 's of the phase space polynomials, one defines the KoszulTate derivative $\delta$ by

$$
\begin{aligned}
\delta q^{i}=0= & \delta p^{i}=0=\delta \eta^{a} \\
\delta \mathcal{P}_{a} & =-C_{a} \\
\operatorname{deg} \delta & =-1
\end{aligned}
$$

then

$$
\begin{aligned}
& H_{0}(\delta)=C^{\infty}(\Sigma) \\
& H_{k}(\delta)=0 \forall k \neq 0
\end{aligned}
$$

It selects all phase space functions regular on the constraint surface.

Second, taking for the grading the degree in the $\eta^{a}$ 's of the phase space polynomials, one defines the longitudinal derivative $d$ by

$$
\begin{aligned}
d F & =\left\{F, C_{a}\right\} \eta^{a} \\
d \eta^{a} & =\frac{1}{2} \eta^{b} \eta^{c} f_{c b}{ }^{a} \\
\text { degd } & =+1
\end{aligned}
$$

then

$$
H^{0}(d)=\{\text { functions invariant on } \Sigma\}
$$


The last step is to show that there exists a differential $s$ defined by

$$
s=\delta+d+\ldots
$$

when the grading equals the difference between the ghost number and the antighost number. Here the ... mean contributions from operators of higher degrees. This differential acts on an arbitrary phase space function in the following way:

$$
s F=\{F, \Omega\}_{E}
$$

The index $E$ stresses the fact that the Poisson bracket is the one generalized to the extended phase space.

$H_{0}(s)$ contains all gauge invariant functions. $\Omega$ is the BRST charge. It is the generator of the BRST symmetry. Since $s^{2}=0$, one gets

$$
\{\Omega, \Omega\}=0
$$

It is a nilpotent operator. This property allows us to compute it.

\section{Computation of the BRST Charge}

In this section, it is shown that the computation of the BRST charge can be made fully algorithmic. Again, for a more detailed explanation the reader is referred elsewher $[4,7]$.

\subsection{The Generalized Poisson Algebra}

To be able to build the algorithm, a restriction must be made on the algebra of first class constraints: it is that the 'structure constants' may only depend on phase space through the constraints themselves, i.e.

$$
\left\{C_{a}, C_{b}\right\}=f_{a b}^{c}(C)
$$

For practical reasons, the $f_{a b}{ }^{c}$ are required to be polynomials in the $C$ 's so that the previous equation may be rewritten

$$
\left\{C_{a}, C_{b}\right\}=\sum_{i=1}^{q} f_{i a b}^{a_{1} \ldots a_{i}} \prod_{j=1}^{i} C_{a_{j}}
$$

where the $f_{i}$ 's are constants and $q$ is an integer.

\subsection{The Algorithm}

The BRST charge can be developed as a sequence over the degrees in $\mathcal{P}_{a}$ 's:

$$
\begin{aligned}
\Omega & =\sum_{n=0}^{N-1} \Omega^{(n)} \\
& =\sum_{n=0}^{N-1} A_{i_{1} \ldots i_{n}+1}^{j_{1} \ldots j_{n}} \eta^{i_{1}} \ldots \eta^{i_{n}+1} \mathcal{P}_{j_{1}} \ldots \mathcal{P}_{j_{n}}
\end{aligned}
$$


with $A \equiv A(C, f) . \Omega$ has parity equal to 1 , as it must be. On the left-hand side, the integer $N$ is not explicitly written as an index since it is not known a priori, whether the right-hand side is a polynomial or not. In case it is a polynomial, the expression (49) furnishes a closed form expression of $\Omega$. It is easily seen, then, that

$$
\Omega^{(0)}=\eta^{a} C_{a}
$$

This is a gauge transformation in which the parameters $\epsilon^{a}$ have been replaced by $\eta^{a}$. If $\mathcal{O}$ is any operator in the graded differential algebra, the differential $\delta$ is such that

$$
\delta(\mathcal{O})=\left\{\mathcal{O}, \Omega^{(0)}\right\}
$$

From this and the nilpotent property of $\Omega(47)$, one can deduce

$$
\delta \Omega^{(n+1)}=-D^{(n)}
$$

where

$$
D^{(n)}=\frac{1}{2}\left(\sum_{k=0}^{n}\left\{\Omega^{(k)}, \Omega^{(n-k)}\right\}_{C}+\sum_{k=0}^{n-1}\left\{\Omega^{(k+1)}, \Omega^{(n-k)}\right\}_{g h}\right)
$$

The index $C$ means that the Poisson bracket is the ordinary bracket taken with respect to the $\left(q^{i}, p_{j}\right)$ while the index $g h$ means that the bracket is given by (22). From (52) one sees that at each stage of the recursive calculation $\Omega^{(n)}$ is determined only up to a $\delta$ exact expression, since $\delta^{2}=0$. Therefore, there is a large degree of indeterminency on the expression of $\Omega$. This is a problem for the algorithmization of the calculation. Therefore, one is to introduce some additional input in order to select a given solution. Two types of restrictions have been tried. The first requires that the number of terms in the expressions $\Omega^{(n)}$ for each $n$ be minimized. Apart from its practical interest for the calculation itself, it has no other motivation. How to do that in an algorithmic way is explained in Burnel et al. [4]. The second is more theoretically grounded. Suppose there exists a grading such that a contracting homotopy $\sigma$ exists. It verifies

$$
(\sigma \delta+\delta \sigma) A_{k}=N_{k} A_{k}
$$

With respect to that grading, one can write (52)

$$
\delta \Omega_{k}^{(n+1)}=-D_{k}^{(n)}
$$

for all $k$. This equation can be rewritten as

$$
\delta \Omega^{(n)}=\delta\left(\sum_{k} \frac{1}{N_{k}} \sigma D_{k}^{(n-1)}\right)
$$

if we one takes (54) into account. The particular solution is obtained if one drops $\delta$ on both sides. One can show that such a $\sigma$ exists for all Poisson algebras obeying the restrictions defined in the previous subsection. It is given by Dresse [7]

$$
\sigma=\frac{\overleftarrow{\partial}}{\partial C_{a}} \mathcal{P}_{a}
$$


The corresponding solution is called the 'covariant' solution because it remains invariant under any regular transformation

$$
\begin{aligned}
& C_{a} \quad \rightarrow \quad M_{a}^{b} C_{b} \\
& \mathcal{P}_{a} \quad \rightarrow \quad M_{a}^{b} \mathcal{P}_{b} \\
& \eta^{a} \quad \rightarrow \quad\left(M^{-1}\right)^{a}{ }_{b} \eta^{b}
\end{aligned}
$$

\section{Applications}

The computation of the BRST charge is trivial if the Poisson algebra is a Lie algebra. This is the case in all usual gauge theories closely linked to present phenomenology. The algorithm of Sect. 4 may be applied in many cases which go beyond the usual gauge theories. However, in this extended context, the calculations are much more complex and very often untractable by hand calculations. One is not even sure that there exists a closed form of $\Omega$. The recursive procedure must be pursued toward very big values of $N$, eventually, to be able to see that. This justifies the use of computer algebra in this context.

\subsection{Implementation}

Implementation has been done in Reduce, and it uses two complementary packages DUUMY [8] and ASS IST [9]. It is written in the symbolic language. A few important remarks are in order:

(a) Since the number of constraints may be anything (and may be large), it is absolutely necessary to avoid an explicit representation of indices. The package DUMMY offers precisely the possibility to work with dummy indices and, therefore, to free oneself from the number of constraints. This package also allows us

(i) to find a canonical form to any expression involving dummy indices,

(ii) to include anticommutative as well as commutative operators, and

(iii) to take into account full and mixed symmetries.

The many reordering operations necessary to achieve these properties made it necessary to base the internal representation on the vector data structure for efficiency reasons. It is one of the functions of ASSIST to allow us to manipulate them in a list-like way.

The restriction on the kind of mixed symmetries which can be considered is that they may be expressed as a tree-like configuration.

(b) The package contains three files. The first contains the part of the algorithm which is application independent. The second contains the basic Poisson brackets; the third contains the Jacobi identities. The inclusion of Jacobi identities is crucial to obtain a compact meaningful result. Of course, when they are involved, we can only get a normal form. When there are several of them, the search for the normal form may be time consuming. It is done by a function which is not automatically called by the main algorithm, but which can be used at each step of the recursive calculation. 


\subsection{Results}

The program has been applied to the usual linear algebra, to several quadratic algebras and to cubic algebras. A full discussion can be found elsewhere $[4,10]$. Here, one considers only the simplest example of the linear algebra, and the most spectacular of the generic cubic algebra which is a generalization of the spin 4 algebra.

\subsubsection{Usual Linear Lie Algebras}

The constraint algebra is given by

$$
\left\{C_{a}, C_{b}\right\}=f_{a b}^{c} C_{c} .
$$

Using the program, one easily recovers the well known result

$$
\Omega^{(1)}=\frac{1}{2} f_{a b}{ }^{c} \eta^{a b} \mathcal{P}_{c}
$$

Computing $D^{(1)}$ and checking that $\delta D^{(1)}=0$ leads to the Jacobi identity

$$
f_{[a b}^{c} f_{d] c}^{e}=0
$$

One gets $D^{(1)}=0$. This stops the construction because all the higher order contributions vanish.

\subsubsection{The Generic Cubic Algebra}

Of course, here there is no reason to find a closed form for $\Omega$. It is for this (rather academic) example that the power of the package can be best appreciated.

The generic Poisson algebra is

$$
\left\{C_{d_{1}}, C_{d_{2}}\right\}=f_{d_{1} d_{2}}^{d_{3}} C_{d_{3}}+D_{d_{1} d_{2}}^{d_{3} d_{4}} C_{d_{3}} C_{d_{4}}+E_{d_{1} d_{2}}^{d_{3} d_{4} d_{5}} C_{d_{3}} C_{d_{4}} C_{d_{5}}
$$

It is seen that the constants $f, D$ and $E$ must satisfy the five Jacobi identities:

$$
\begin{aligned}
& f_{[a b}^{c} f_{d] c}^{e}=0, \\
& f_{[a b}^{c} d_{d] c}^{e f}+D_{[a b}^{c e} f_{d] c}^{f}+D_{[a b}^{c f} f_{d] c}^{e}=0, \\
& 2 D_{d_{7}\left[d_{4}\right.}^{\left\{d_{1} d_{2}\right.} D_{\left.d_{5} d_{6}\right]}^{\left.d_{3}\right\} d_{7}}+E_{d_{7}\left[d_{4}\right.}^{\left\{d_{1} d_{2} d_{3}\right\}} f_{\left.d_{5} d_{6}\right]}^{d_{7}}+3 f_{d_{7}\left[d_{4}\right.}^{\left\{d_{1}\right.} E_{\left.d_{5} d_{6}\right]}^{\left.d_{2} d_{3}\right\} d_{7}}=0 \text {, } \\
& 3 D_{d_{1}\left[d_{2}\right.}^{\left\{d_{3} d_{4}\right.} E_{\left.d_{7} d_{8}\right]}^{\left.d_{5} d_{6}\right\} d_{1}}+2 E_{d_{1}\left[d_{2}\right.}^{\left\{d_{3} d_{4} d_{5}\right.} D_{\left.d_{7} d_{8}\right]}^{\left.d_{6}\right\} d_{1}}=0, \\
& E_{\left[d_{6} d_{7}\right.}^{d_{8}\left\{d_{1} d_{2}\right.} E_{\left.d_{9}\right] d_{8}}^{\left.d_{3} d_{4} d_{5}\right\}}=0 .
\end{aligned}
$$

The calculation order by order can be done. The result is explosive if one does not enforce the simplification induced by the above identities. The one and six order results are

$$
\Omega^{(1)}=\left(\eta^{d_{1}} \eta^{d_{2}} \mathcal{P}_{d_{3}}\left(f_{d_{1} d_{2}}^{d_{3}}+D_{d_{1} d_{2}}^{d_{4} d_{3}} C_{d_{4}}+E_{d_{1} d_{2}}^{d_{4} d_{5} d_{3}} C_{d_{4}} C_{d_{5}}\right)\right) / 2
$$




$$
\begin{aligned}
& \Omega^{(6)}=\left(\eta^{d_{1}} \eta^{d_{2}} \eta^{d_{3}} \eta^{d_{4}} \eta^{d_{5}} \eta^{d_{6}} \eta^{d_{7}} \mathcal{P}_{d_{8}} \mathcal{P}_{d_{9}} \mathcal{P}_{d_{10}} \mathcal{P}_{d_{11}} \mathcal{P}_{d_{12}} \mathcal{P}_{d_{13}}\right. \\
& \left(864 f_{d_{14} d_{2}}^{d_{9}} f_{d_{15} d_{16}}^{d_{10}} f_{d_{17} d_{18}}^{d_{11}} C_{d_{19}} E_{d_{3} d_{4}}^{d_{15} d_{17} d_{12}} E_{d_{5} d_{6}}^{d_{18} d_{19} d_{13}}\right. \\
& E_{d_{7} d_{1}}^{d_{14} d_{16} d_{8}}+864 f_{d_{14} d_{15}}^{d_{9}} f_{d_{16} d_{2}}^{d_{10}} f_{d_{17} d_{18}}^{d_{11}} C_{d_{19}} E_{d_{3} d_{4}}^{d_{17} d_{19} d_{12}} \\
& E_{d_{5} d_{6}}^{d_{14} d_{16} d_{13}} E_{d_{7} d_{1}}^{d_{15} d_{18} d_{8}}-126 f_{d_{14} d_{15}}^{d_{9}} f_{d_{16} d_{17}}^{d_{10}} D_{d_{3} d_{4}}^{d_{18} d_{12}} \\
& D_{d_{18} d_{2}}^{d_{16} d_{11}} C_{d_{19}} E_{d_{5} d_{6}}^{d_{14} d_{17} d_{13}} E_{d_{7} d_{1}}^{d_{15} d_{19} d_{8}}-63 f_{d_{14} d_{15}}^{d_{9}} D_{d_{2} d_{3}}^{d_{16} d_{10}} \\
& D_{d_{4} d_{5}}^{d_{17} d_{11}} D_{d_{16} d_{17}}^{d_{18} d_{12}} D_{d_{18} d_{6}}^{d_{14} d_{13}} C_{d_{19}} E_{d_{7} d_{1}}^{d_{15} d_{19} d_{8}}-42 f_{d_{14} d_{15}}^{d_{9}} \\
& D_{d_{2} d_{3}}^{d_{16} d_{10}} D_{d_{4} d_{5}}^{d_{18} d_{11}} D_{d_{16} d_{6}}^{d_{17} d_{12}} D_{d_{18} d_{17}}^{d_{14} d_{13}} C_{d_{19}} E_{d_{7} d_{1}}^{d_{15} d_{19} d_{8}}-168 \\
& f_{d_{14} d_{15}}^{d_{9}} D_{d_{2} d_{3}}^{d_{16} d_{10}} D_{d_{16} d_{4}}^{d_{17} d_{11}} D_{d_{17} d_{5}}^{d_{18} d_{12}} D_{d_{18} d_{6}}^{d_{11} d_{13}} C_{d_{19}} E_{d_{7} d_{1}}^{d_{15} d_{19} d_{8}} \\
& +84 D_{d_{1} d_{2}}^{d_{14} d_{11}} D_{d_{3} d_{4}}^{d_{17} d_{12}} D_{d_{5} d_{6}}^{d_{18} d_{13}} D_{d_{14} d_{15}}^{d_{16} d_{10}} D_{d_{17} d_{18}}^{d_{19} d_{9}} D_{d_{19} d_{7}}^{d_{15} d_{8}} C_{d_{16}} \\
& +56 D_{d_{1} d_{2}}^{d_{14} d_{11}} D_{d_{3} d_{4}}^{d_{17} d_{12}} D_{d_{5} d_{6}}^{d_{19} d_{13}} D_{d_{14} d_{15}}^{d_{16} d_{10}} D_{d_{17} d_{18}}^{d_{15} d_{9}} D_{d_{19} d_{7}}^{d_{18} d_{8}} C_{d_{16}} \\
& +168 D_{d_{1} d_{2}}^{d_{17} d_{11}} D_{d_{3} d_{4}}^{d_{18} d_{12}} D_{d_{5} d_{6}}^{d_{19} d_{13}} D_{d_{14} d_{15}}^{d_{16} d_{10}} D_{d_{17} d_{18}}^{d_{15} d_{9}} D_{d_{19} d_{7}}^{d_{14} d_{8}} C_{d_{16}} \\
& +224 D_{d_{2} d_{3}}^{d_{14} d_{10}} D_{d_{4} d_{5}}^{d_{17} d_{11}} D_{d_{14} d_{15}}^{d_{16} d_{9}} D_{d_{17} d_{1}}^{d_{18} d_{8}} D_{d_{18} d_{7}}^{d_{19} d_{13}} D_{d_{19} d_{6}}^{d_{15} d_{12}} C_{d_{16}} \\
& +84 D_{d_{2} d_{3}}^{d_{16} d_{11}} D_{d_{4} d_{5}}^{d_{17} d_{12}} D_{d_{14} d_{1}}^{d_{15} d_{10}} D_{d_{16} d_{17}}^{d_{18} d_{9}} D_{d_{18} d_{7}}^{d_{19} d_{8}} D_{d_{19} d_{6}}^{d_{14} d_{13}} \\
& C_{d_{15}}-126 D_{d_{2} d_{3}}^{d_{16} d_{11}} D_{d_{4} d_{5}}^{d_{18} d_{12}} D_{d_{6} d_{7}}^{d_{19} d_{13}} D_{d_{14} d_{1}}^{d_{15} d_{10}} D_{d_{16} d_{17}}^{d_{14} d_{8}} \\
& D_{d_{18} d_{19}}^{d_{17} d_{9}} C_{d_{15}}-140 D_{d_{2} d_{3}}^{d_{16} d_{11}} D_{d_{4} d_{5}}^{d_{18} d_{12}} D_{d_{14} d_{1}}^{d_{15} d_{10}} D_{d_{16} d_{6}}^{d_{17} d_{13}} \\
& D_{d_{17} d_{19}}^{d_{11} d_{9}} D_{d_{18} d_{7}}^{d_{19} d_{8}} C_{d_{15}}+336 D_{d_{2} d_{3}}^{d_{16} d_{11}} D_{d_{4} d_{5}}^{d_{18} d_{12}} D_{d_{14} d_{10}}^{d_{15} d_{10}} \\
& D_{d_{16} d_{17}}^{d_{14} d_{9}} D_{d_{18} d_{7}}^{d_{19} d_{8}} D_{d_{19} d_{6}}^{d_{17} d_{13}} C_{d_{15}}+56 D_{d_{2} d_{3}}^{d_{16} d_{11}} D_{d_{4} d_{5}}^{d_{19} d_{12}} \\
& D_{d_{14} d_{1}}^{d_{15} d_{10}} D_{d_{16} d_{17}}^{d_{18} d_{9}} D_{d_{18} d_{6}}^{d_{14} d_{13}} D_{d_{19} d_{7}}^{d_{17} d_{8}} C_{d_{15}}-56 D_{d_{2} d_{3}}^{d_{17} d_{10}} D_{d_{4} d_{5}}^{d_{18} d_{11}} \\
& D_{d_{14} d_{15}}^{d_{16} d_{9}} D_{d_{17} d_{7}}^{d_{15} d_{13}} D_{d_{18} d_{1}}^{d_{19} d_{8}} D_{d_{19} d_{6}}^{d_{14} d_{12}} C_{d_{16}}-224 D_{d_{3} d_{4}}^{d_{16} d_{10}} \\
& \left.\left.D_{d_{14} d_{2}}^{d_{15} d_{9}} D_{d_{16} d_{1}}^{d_{17} d_{8}} D_{d_{17} d_{7}}^{d_{19} d_{13}} D_{d_{18} d_{5}}^{d_{14} d_{11}} D_{d_{19} d_{6}}^{d_{18} d_{12}} C_{d_{15}}\right)\right) / 211680
\end{aligned}
$$

Some mathematical questions can be raised at this point:

- What are the conditions required on the structure constants to guarantee the existence of a closed form solution for $\Omega$ ? What are their meanings?

- Among the many solutions, the most concise (i.e. the one which is of lowest order in $\mathcal{P}_{a}$ ) is not, in general, the covariant one. So the minimal rank of the algebra is difficult to determine.

Some considerations on the above questions have already been made: $[4,7,10]$. To properly answer these questions, however, a deeper study of nonlinear Poisson algebras should be made. No doubt the computation of the BRST charge can play a role in that study. The package BRST turns out to be potentially very useful in that context. 


\section{Acknowledgements}

This work has been supported by the Fonds National de la Recherche Scientifique (FNRS) and by INTAS contract 93-0030.

\section{References}

[1] Becchi, C., Rouet, A. and Stora, R. (1976). Commun. Math. Phys. 42, 127; Ann. Phys. (N.Y.) 98, 287.

[2] Tyutin, I. V. (1975). Lebedev preprint FIAN 39.

[3] Henneaux, M. and Teitelboim, C. (1992). Quantization of Gauge Systems. Princeton University Press.

[4] Burnel, A., Caprasse, H. and Dresse, A. (1994). Int. J. MPC 5(6), 1035.

[5] Dirac, P. A. M. Lectures on Quantum Mechanics. Yeshiva University, Academic Press, New York.

[6] 't Hooft, G. (1994). Gauge Theory and Renormalization. Int. Conf. 'The History of Original Ideas and Basic Discoveries in Particle Physics', Erice, Italy.

[7] Dresse, A. (1994). PhD thesis, ULB.

[8] Dresse, A. (1994). DUMMY . RED REDUCE library.

[9] Caprasse, H. (1993.) ASSIST . RED REDUCE library.

[10] Dresse, A. and Henneaux, M. (1994). J. Math. Phys. 35(3), 1334. 